$\xi=$

\title{
To unite nuclear and sub-nuclear strong interactions
}

\author{
U. V. S. Seshavatharam ${ }^{1} *$, S. Lakshminarayana ${ }^{2}$ \\ ${ }^{1}$ Honorary Faculty, I-SERVE, Survey no-42, Hitex road, Hitech city, Hyderabad-84, Telangana, India \\ ${ }^{2}$ Department of Nuclear Physics, Andhra University, Visakhapatnam-03, AP, India \\ *Corresponding authorE-mail: Seshavatharam.uvs@gmail.com
}

\begin{abstract}
With reference to 'reciprocal' of the strong coupling constant and 'reduced Compton's wavelength' of the nucleon, we make an attempt to understand the background of nuclear charge radius, binding energy and stability.
\end{abstract}

Keywords: Strong Coupling Constant; Reduced Compton Wavelength of Nucleon; Beta Stability Line; Semi-Empirical Mass Formula (SEMF).

\section{Introduction}

The modern theory of strong interaction is quantum chromo dynamics (QCD) [1]. It explores baryons and mesons in broad view with 6 quarks and 8 gluons. According to QCD, the four important properties of strong interaction are: 1) color charge; 2) confinement; 3) asymptotic freedom [2]; 4) short-range nature $\left(<10^{-15} \mathrm{~m}\right)$. Color charge is assumed to be responsible for the strong force to act on quarks via the force carrying agent, gluon. Experimentally it is well established that, strength of strong force depends on the energy through the interaction or the distance between particles. At lower energies or longer distances: a) color charge strength increase; b) strong force becomes 'stronger'; c) nucleons can be considered as fundamental nuclear particles and quarks seem to be strongly bound within the nucleons leading to 'Quark confinement'. At high energies or short distances: a) color charge strength decreases; b) strong force gets 'weaker';3) colliding protons generate 'scattered free quarks leading to 'Quark Asymptotic freedom'. Based on these points, low energy nuclear scientists assume 'strong interaction' as a strange nuclear interaction associated with binding of nucleons and implications and its implications were not considered. High-energy nuclear scientists consider nucleons as composite states of quarks and try to understand the nature and strength of strong interaction at sub nuclear level.

At this juncture, one important question to be answered and reviewed at the basic level is: How to understand nuclear interactions in terms of sub nuclear interactions? Unfortunately, the famous nuclear models like, Liquid drop model and Fermi's gas model [3-6] are lagging in answering this question. To find a way, we would like to suggest that, by implementing the 'strong coupling constant' $\left(\alpha_{s} \cong 0.1186\right)$, in low energy nuclear physics, nuclear binding energy and stability can be understood. In this new direction, we have developed interesting concepts and produced many semi empirical relations [7-9]. Even though it is in its budding stage, our model seems to be simple and realistic compared to the new integrated model proposed by N. Ghahramany et al [10-12]. It needs further study at a fundamental level.

\section{Role of the strong coupling constant in low energy nuclear physics}

We propose the following four assumptions.

1) Nuclear binding energy can be understood with a single energy coefficient associated with 'reciprocal' of the strong coupling constant.

2) Characteristic nuclear radius can be expressed as, $R_{0} \cong\left(\frac{1}{\sqrt{\alpha_{s}}}\right)\left\{\frac{}{m_{p} c}+\frac{}{m_{n} c}\right\} \cong\left(\frac{1}{\sqrt{\alpha_{s}}}\right)\left(\frac{2}{m_{p} c}\right) \cong 1.22 \mathrm{fm}$.

3) Characteristic nuclear binding energy potential can be, $B_{0} \cong\left(\frac{1}{\alpha_{s}}\right) \frac{e^{2}}{4 \pi \varepsilon_{0} R_{0}} \cong\left(\frac{\alpha}{2 \sqrt{\alpha_{s}}}\right) m_{p} c^{2} \cong 10.0 \mathrm{MeV}$ where $R_{0} \approx 1.22 \mathrm{fm}$.

4) $\left(\alpha_{s}\right)$ and $\exp \left(\frac{m_{n}-m_{p}}{m_{e}}\right) \cong 4 \pi$ Seem to play a crucial role in understanding beta stability line and nuclear binding energy.

\section{About the semi empirical mass formula}

Let $A$ be the total number of nucleons, $Z$ the number of protons and $N$ the number of neutrons. According to the semi-empirical mass formula (SEMF), nuclear binding energy:

$B=a_{v} A-a_{s} A^{2 / 3}-a_{c} \frac{Z(Z-1)}{A^{1 / 3}}-a_{a} \frac{(A-2 Z)^{2}}{A} \pm \frac{a_{p}}{\sqrt{A}}$

Here $a_{v}=$ volume energy coefficient, $a_{s}$ is the surface energy coefficient, $a_{c}$ is the coulomb energy coefficient, $a_{a}$ is the asymmetry energy coefficient and $a_{p}$ is the pairing energy coefficient. If we consider the sum of the volume energy, surface energy, coulomb energy, asymmetry energy and pairing energy, then the picture of a nucleus as a drop of incompressible liquid roughly accounts for the observed variation of binding energy of the nucleus. 
By maximizing $B(A, Z)$ with respect to $Z$, one can find the number of protons $Z$ of the stable nucleus of atomic weight $A$ as,

$$
Z \approx \frac{A}{2+\left(a_{c} / 2 a_{a}\right) A^{2 / 3}} \text { and } A-2 Z \approx \frac{0.4 A^{2}}{A+200}
$$

By substituting the above value of $Z$ back into $B$ one obtains the binding energy as a function of the atomic weight, $B(A)$.

Maximizing $B(A) / A$ with respect to $\mathrm{A}$ gives the nucleus which is most strongly bound or most stable.

\section{Beta stability line with respect to strong coupling constant}

If $\alpha_{s} \cong 0.1186$, for $Z>8$, close to the line of beta stability, for $Z>8$,

$A_{s} \cong\left(Z+\sqrt{\frac{1}{\alpha_{s}}}\right)^{6 / 5} \cong(Z+2.904)^{1.2}$

$$
\left.\begin{array}{l}
\text { for } Z>16 \\
\left(A_{s}-2 Z\right) \cong(Z \beta+1)^{2}-4 \\
\text { where } \beta=\left(\frac{3}{5}\right) \alpha_{s} .
\end{array}\right\}
$$

\section{Beta stability line with respect to nucleon mass difference}

With reference to nucleon and electron rest masses, we noticed that,

$\exp \left(\frac{\left(m_{n}-m_{p}\right) c^{2}}{m_{e} c^{2}}\right) \cong 12.5659102 \cong 4 \pi$

where, $m_{n} c^{2} \cong 939.565413 \mathrm{MeV}$,

$\left\{m_{p} c^{2} \cong 938.272081 \mathrm{MeV} ; m_{e} c^{2} \cong 0.5109989461 \mathrm{MeV}\right.$

Based on this observation, beta stability line can be understood with the following empirical relations.

$$
\text { Let, } k \cong(1 / 4 \pi)^{2} \cong 0.006333
$$

For $\mathrm{Z} \geq 2$,

$A_{s} \cong 2 Z+(1 / 4 \pi)^{2} \cong 2 Z+0.006333 Z^{2} \cong 2 Z+k Z^{2}$

$N_{s} \cong Z+(1 / 4 \pi)^{2} \cong Z+0.006333 Z^{2} \cong Z+k Z^{2}$

$A_{s}-2 Z \cong(1 / 4 \pi)^{2} \cong k Z^{2}$ and $\frac{Z}{\sqrt{A_{s}-2 Z}} \cong 4 \pi$

Based on these relations,
A) $\frac{\left(A_{s}-2 Z\right)^{2}}{A} \cong k^{2} A_{s}\left(A_{s}-Z\right) \sqrt{Z}$
B) $\frac{A_{s}^{1 / 2}\left(A_{s}-Z\right)^{1 / 4} Z^{1 / 8}}{\sqrt{A_{s}-2 Z}} \approx \frac{1}{\sqrt{k}} \approx 4 \pi$

\section{Relations for understanding nuclear bind- ing energy}

Based on the new integrated model proposed by N. Ghahramany et al [10-12] and with reference to relation (7), it is possible to show that, for $Z \cong(40$ to 83$)$, close to the beta stability line,

$\left[\frac{N_{s}^{2}-Z^{2}}{Z}\right] \cong k Z A$

Based on this strange and simple relation and with reference to our recent publications [8], [9], close to the beta stability line, numerically it is possible to show that, for $(Z \geq 24)$, if $\alpha_{s} \approx 0.1186$ and $R_{0} \approx 1.22 \mathrm{fm}$,

$(B)_{A} \cong\left[A-A^{1 / 3}-\frac{k A \sqrt{N Z}}{3.4}\right] \times 10.0 \mathrm{MeV}$

$\left.\cong\left\{A-A^{1 / 3}-\left[\left(\alpha_{s}\right)\left(\frac{a_{c}}{2 a_{a}}\right)(A \sqrt{N Z})\right]\right\} \times 10.0 \mathrm{MeV}\right\}$

where, $\alpha_{s}\left(\frac{a_{c}}{2 a_{a}}\right) \cong \frac{k}{3.4}$. It is for further study. For $(Z \geq 24)$, binding energy per nucleon can be expressed as:

$\frac{(B)_{A}}{A} \cong\left[1-A^{-2 / 3}-\frac{k \sqrt{N Z}}{3.4}\right] \times 10.0 \mathrm{MeV}$

a) See table 1 for nuclear binding energy of stable and unstable isotopes of $Z=23$ to 100 estimated from relations (7) and (10).

b) See table 2 for nuclear binding energy of natural isotopes of $\mathrm{Z}=30,40,50,60,70,80$ and 92 estimated from relation (10)

c) See table 3 for nuclear binding energy of natural isotopes of

\begin{tabular}{|c|c|c|c|c|c|}
\hline $\begin{array}{l}\text { Proton } \\
\text { number }\end{array}$ & $\begin{array}{l}\text { Mass } \\
\text { number }\end{array}$ & $\begin{array}{l}\text { Neutron } \\
\text { number }\end{array}$ & $\begin{array}{l}\text { Estimated } \\
\text { binding energy }(\mathrm{MeV})\end{array}$ & $\begin{array}{l}\text { Actual[10] or reference [4] } \\
\text { binding energy }(\mathrm{MeV})\end{array}$ & $\begin{array}{l}\text { Error } \\
(\mathrm{MeV})\end{array}$ \\
\hline 23 & 49 & 26 & 431.1 & 426.34 & -4.7 \\
\hline 24 & 52 & 28 & 457.6 & 456.349 & -1.2 \\
\hline 26 & 56 & 30 & 492.6 & 492.258 & -0.4 \\
\hline 27 & 59 & 32 & 518.8 & 517.313 & -1.5 \\
\hline 28 & 61 & 33 & 536.1 & 534.666 & -1.4 \\
\hline 29 & 63 & 34 & 553.4 & 551.385 & -2.0 \\
\hline 31 & 68 & 37 & 596.3 & 590.61 & -5.7 \\
\hline 32 & 70 & 38 & 613.3 & 610.521 & -2.8 \\
\hline 33 & 73 & 40 & 638.8 & 634.34 & -4.5 \\
\hline 34 & 75 & 41 & 655.7 & 651.02 & -4.7 \\
\hline 35 & 78 & 43 & 680.9 & 676.11 & -4.8 \\
\hline 36 & 80 & 44 & 697.6 & 695.434 & -2.2 \\
\hline
\end{tabular}
$Z=25$ to 83 estimated from relation (10).

Table 1: Nuclear Binding Energy of $Z=23$ To 100 Estimated From Relations (7) and (10) 


\begin{tabular}{|c|c|c|c|c|c|}
\hline 37 & 83 & 46 & 722.6 & 720.46 & -2.1 \\
\hline 38 & 85 & 47 & 739.1 & 737.85 & -1.3 \\
\hline 39 & 88 & 49 & 763.9 & 763.88 & 0.0 \\
\hline 40 & 90 & 50 & 780.2 & 783.893 & 3.7 \\
\hline 41 & 93 & 52 & 804.7 & 805.765 & 1.1 \\
\hline 42 & 95 & 53 & 820.9 & 821.625 & 0.7 \\
\hline 43 & 98 & 55 & 845.1 & 844.4 & -0.7 \\
\hline 44 & 100 & 56 & 861.1 & 861.928 & 0.8 \\
\hline 45 & 103 & 58 & 885.1 & 884.163 & -0.9 \\
\hline 46 & 105 & 59 & 900.9 & 899.914 & -1.0 \\
\hline 47 & 108 & 61 & 924.7 & 922.2 & -2.5 \\
\hline 48 & 111 & 63 & 948.2 & 947.622 & -0.6 \\
\hline 49 & 113 & 64 & 963.8 & 963.094 & -0.7 \\
\hline 50 & 116 & 66 & 987.1 & 988.684 & 1.6 \\
\hline 51 & 118 & 67 & 1002.5 & 1000.48 & -2.0 \\
\hline 52 & 121 & 69 & 1025.5 & 1024.43 & -1.1 \\
\hline 53 & 124 & 71 & 1048.5 & 1046.32 & -2.1 \\
\hline 54 & 126 & 72 & 1063.5 & 1063.909 & 0.4 \\
\hline 55 & 129 & 74 & 1086.2 & 1085.08 & -1.1 \\
\hline 56 & 132 & 76 & 1108.7 & 1110.038 & 1.4 \\
\hline 57 & 135 & 78 & 1131.0 & 1131.00 & 0.0 \\
\hline 58 & 137 & 79 & 1145.7 & 1145.7 & 0.0 \\
\hline 59 & 140 & 81 & 1167.8 & 1168.67 & 0.9 \\
\hline 60 & 143 & 83 & 1189.7 & 1191.266 & 1.5 \\
\hline 61 & 146 & 85 & 1211.5 & 1209.52 & -2.0 \\
\hline 62 & 148 & 86 & 1225.8 & 1225.392 & -0.4 \\
\hline 63 & 151 & 88 & 1247.3 & 1244.141 & -3.2 \\
\hline 64 & 154 & 90 & 1268.7 & 1266.627 & -2.1 \\
\hline 65 & 157 & 92 & 1289.9 & 1287.38 & -2.5 \\
\hline 66 & 160 & 94 & 1311.0 & 1309.455 & -1.5 \\
\hline 67 & 162 & 95 & 1324.7 & 1321.18 & -3.6 \\
\hline 68 & 165 & 97 & 1345.5 & 1343.08 & -2.5 \\
\hline 69 & 168 & 99 & 1366.2 & 1363.31 & -2.9 \\
\hline 70 & 171 & 101 & 1386.7 & 1384.744 & -1.9 \\
\hline 71 & 174 & 103 & 1407.0 & 1404.44 & -2.6 \\
\hline 72 & 177 & 105 & 1427.2 & 1425.185 & -2.0 \\
\hline 73 & 180 & 107 & 1447.2 & 1444.663 & -2.6 \\
\hline 74 & 183 & 109 & 1467.1 & 1465.525 & -1.6 \\
\hline 75 & 186 & 111 & 1486.8 & 1484.63 & -2.2 \\
\hline 76 & 189 & 113 & 1506.4 & 1505.007 & -1.4 \\
\hline 77 & 192 & 115 & 1525.8 & 1523.81 & -2.0 \\
\hline 78 & 195 & 117 & 1545.0 & 1545.682 & 0.7 \\
\hline 79 & 198 & 119 & 1564.1 & 1564.94 & 0.8 \\
\hline 80 & 201 & 121 & 1583.1 & 1587.411 & 4.3 \\
\hline 81 & 204 & 123 & 1601.9 & 1606.87 & 5.0 \\
\hline 82 & 207 & 125 & 1620.5 & 1629.063 & 8.6 \\
\hline 83 & 210 & 127 & 1639.0 & 1643.94 & 5.0 \\
\hline 84 & 213 & 129 & 1657.3 & 1659.72 & 2.4 \\
\hline 85 & 216 & 131 & 1675.4 & 1673.42 & -2.0 \\
\hline 86 & 219 & 133 & 1693.5 & 1690.59 & -2.9 \\
\hline 87 & 222 & 135 & 1711.3 & 1706.49 & -4.8 \\
\hline 88 & 225 & 137 & 1729.0 & 1724.18 & -4.8 \\
\hline 89 & 228 & 139 & 1746.6 & 1740.67 & -5.9 \\
\hline 90 & 231 & 141 & 1763.9 & 1759.14 & -4.8 \\
\hline 91 & 234 & 143 & 1781.2 & 1776.08 & -5.1 \\
\hline 92 & 238 & 146 & 1804.2 & 1801.69 & -2.6 \\
\hline 93 & 241 & 148 & 1821.1 & 1817.31 & -3.8 \\
\hline 94 & 244 & 150 & 1837.8 & 1835.45 & -2.4 \\
\hline 95 & 247 & 152 & 1854.4 & 1851.73 & -2.7 \\
\hline 96 & 250 & 154 & 1870.8 & 1868.97 & -1.8 \\
\hline 97 & 254 & 157 & 1892.8 & 1888.79 & -4.0 \\
\hline 98 & 257 & 159 & 1908.9 & 1906.19 & -2.7 \\
\hline 99 & 260 & 161 & 1924.8 & 1922.2 & -2.6 \\
\hline 100 & 263 & 163 & 1940.5 & 1939.52 & -1.0 \\
\hline
\end{tabular}

Table 2: Nuclear Binding Energy of Natural Isotopes of $Z=30,40,50,60,70,80,92$ Estimated from Relation (10)

\begin{tabular}{|c|c|c|c|c|c|}
\hline $\begin{array}{l}\text { Proton } \\
\text { number }\end{array}$ & $\begin{array}{l}\text { Mass } \\
\text { number }\end{array}$ & $\begin{array}{l}\text { Neutron } \\
\text { number }\end{array}$ & $\begin{array}{l}\text { Estimated binding energy } \\
(\mathrm{MeV})\end{array}$ & $\begin{array}{l}\text { Actual [10] binding energy } \\
(\mathrm{MeV})\end{array}$ & $\begin{array}{l}\text { Error } \\
(\mathrm{MeV})\end{array}$ \\
\hline 30 & 64 & 34 & 561.9 & 559.098 & -2.83 \\
\hline 30 & 66 & 36 & 579.2 & 578.136 & -1.05 \\
\hline 30 & 68 & 38 & 596.4 & 595.387 & -1.03 \\
\hline 30 & 70 & 40 & 613.6 & 611.087 & -2.53 \\
\hline 40 & 90 & 50 & 780.2 & 783.893 & 3.68 \\
\hline 40 & 91 & 51 & 788.5 & 791.087 & 2.62 \\
\hline 40 & 92 & 52 & 796.7 & 799.722 & 3.02 \\
\hline 40 & 94 & 54 & 813.2 & 814.677 & 1.52 \\
\hline 40 & 96 & 56 & 829.6 & 828.996 & -0.59 \\
\hline 50 & 112 & 62 & 955.6 & 953.532 & -2.11 \\
\hline 50 & 114 & 64 & 971.4 & 971.574 & 0.18 \\
\hline
\end{tabular}




\begin{tabular}{|c|c|c|c|c|c|}
\hline 50 & 115 & 65 & 979.3 & 979.121 & -0.13 \\
\hline 50 & 116 & 66 & 987.1 & 988.684 & 1.58 \\
\hline 50 & 117 & 67 & 995.0 & 995.627 & 0.67 \\
\hline 50 & 118 & 68 & 1002.8 & 1004.955 & 2.16 \\
\hline 50 & 119 & 69 & 1010.6 & 1011.438 & 0.82 \\
\hline 50 & 120 & 70 & 1018.4 & 1020.546 & 2.11 \\
\hline 50 & 122 & 72 & 1034.1 & 1035.53 & 1.47 \\
\hline 50 & 124 & 74 & 1049.6 & 1049.963 & 0.32 \\
\hline 60 & 142 & 82 & 1182.3 & 1185.142 & 2.84 \\
\hline 60 & 144 & 84 & 1197.2 & 1199.083 & 1.92 \\
\hline 60 & 145 & 85 & 1204.6 & 1204.838 & 0.25 \\
\hline 60 & 146 & 86 & 1212.0 & 1212.403 & 0.41 \\
\hline 60 & 148 & 88 & 1226.8 & 1225.028 & -1.76 \\
\hline 60 & 150 & 90 & 1241.6 & 1237.448 & -4.11 \\
\hline 70 & 168 & 98 & 1365.6 & 1362.793 & -2.85 \\
\hline 70 & 170 & 100 & 1379.7 & 1378.13 & -1.55 \\
\hline 70 & 171 & 101 & 1386.7 & 1384.744 & -1.94 \\
\hline 70 & 173 & 103 & 1400.7 & 1399.131 & -1.53 \\
\hline 70 & 174 & 104 & 1407.6 & 1406.595 & -1.05 \\
\hline 70 & 176 & 106 & 1421.6 & 1419.283 & -2.29 \\
\hline 80 & 196 & 116 & 1550.2 & 1551.218 & 1.00 \\
\hline 80 & 198 & 118 & 1563.4 & 1566.489 & 3.10 \\
\hline 80 & 199 & 119 & 1570.0 & 1573.153 & 3.20 \\
\hline 80 & 200 & 120 & 1576.5 & 1581.181 & 4.66 \\
\hline 80 & 201 & 121 & 1583.1 & 1587.411 & 4.34 \\
\hline 80 & 202 & 122 & 1589.6 & 1595.165 & 5.55 \\
\hline 80 & 204 & 124 & 1602.7 & 1608.652 & 5.98 \\
\hline 92 & 234 & 142 & 1780.2 & 1778.567 & -1.63 \\
\hline 92 & 235 & 143 & 1786.2 & 1783.864 & -2.36 \\
\hline 92 & 238 & 146 & 1804.2 & 1801.69 & -2.56 \\
\hline
\end{tabular}

Table 3: Nuclear Binding Energy of Natural Isotopes of $Z=25$ To83 Estimated from Relation (10)

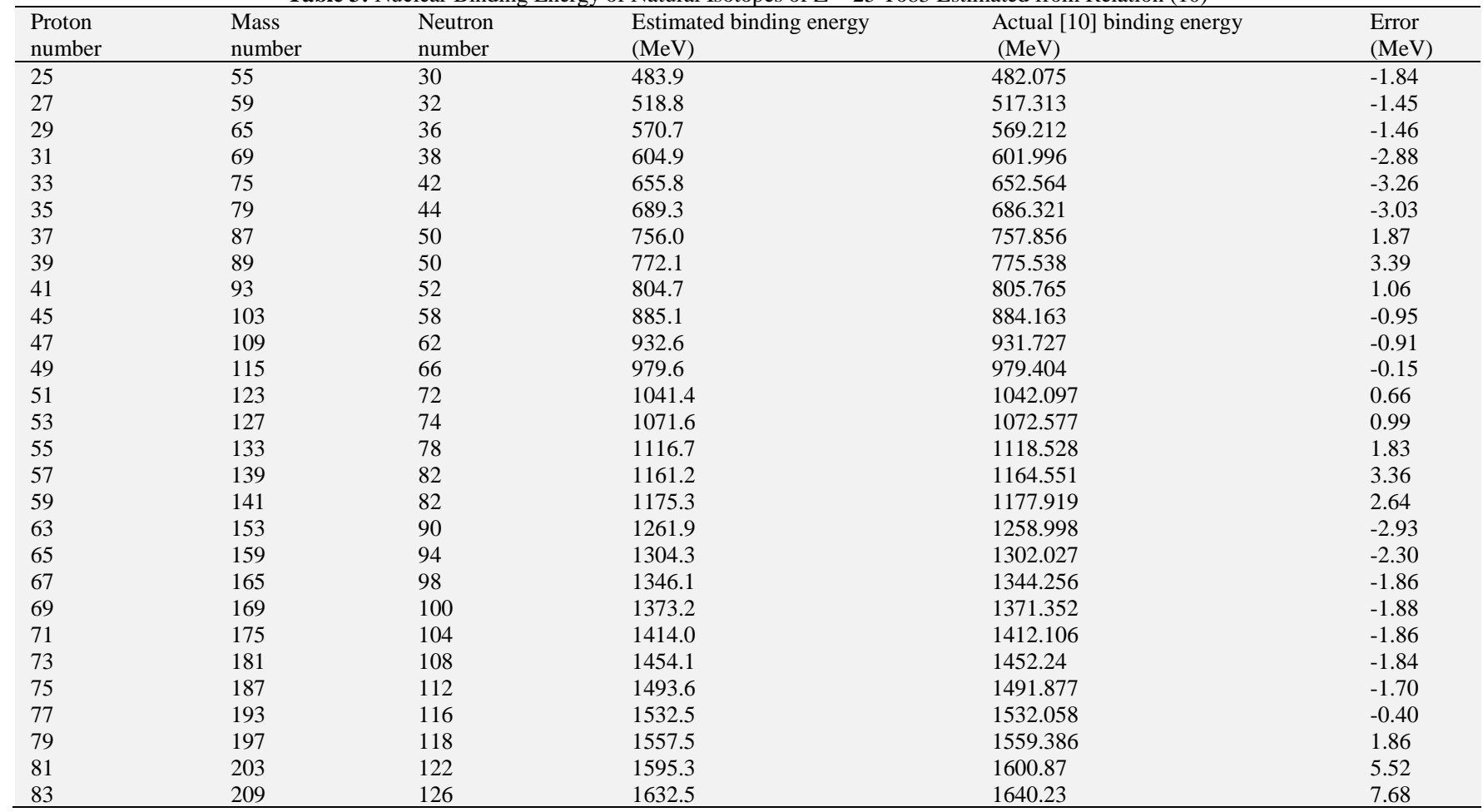

With an error bar of $5 \mathrm{MeV}$, to some extent, relation (10) can be applied to light atomic nuclides and for $\mathrm{Z}=3$ to 100 , modified relation can be expressed as,

$$
(B)_{A} \cong\left[A-A^{1 / 3}-\frac{k A \sqrt{N Z}}{3.35}-1\right] \times 10.09 \mathrm{MeV}
$$

Estimated data can be compared with first four terms of the semi empirical mass formula.

\section{Discussion}

a) By considering coulombic repulsions and with reference to our earlier publications [7], [8], close to the beta stability line, binding energy can also be expressed with: for $(Z \geq 24)$,

$(B)_{A} \cong\left[A-k N\left(\frac{k A A_{s}}{\sqrt{Z}}+1\right)\right] \times 8.9 \mathrm{MeV}$ 
where $\left\{\begin{array}{l}R_{0} \cong 1.22 \mathrm{fm} \text { and } \\ \left\{\left[\left(\frac{1}{\alpha_{s}}\right) \frac{e^{2}}{4 \pi \varepsilon_{0} R_{0}}\right]-\left[\frac{e^{2}}{4 \pi \varepsilon_{0} R_{0}}\right]\right\} \cong 8.9 \mathrm{MeV}\end{array}\right.$

b) By fine tuning the values of $\left(\alpha_{s}\right.$ and $\left.R_{0}\right)$ and by considering even-odd corrections and shell corrections, accuracy can be improved.

c) With a suitable mathematical relation and with a single energy coefficient lying in between (8.9 to 10 ) MeV, it is possible to estimate nuclear binding energy.

d) Close to the beta stability line, ignoring the pairing energy term, semi empirical mass formula energy coefficients can be expressed as: $a_{v} \approx a_{s} \approx a_{a} \approx \frac{3}{2} \times 10.0 \mathrm{MeV} \approx 15.0 \mathrm{MeV}$ and $a_{c} \approx 0.73 \mathrm{MeV}$.

\section{Conclusion}

Understanding and estimating nuclear binding energy and stability with 'sub-nuclear strong interaction' seems to be quite interesting and needs a serious consideration at a fundamental level. We believe that, results obtained from above relations are simple to understand and seem to be more physical and relatively closer to the experimental data. With further research, current nuclear models and strong interaction concepts, pertaining to high-energy physics can be studied in a unified manner, and a realistic nuclear model can be developed.

\section{Acknowledgements}

Author Seshavatharam is indebted to professors brahmashri M. Nagaphani Sarma, Chairman, shri K.V. Krishna Murthy, former Chairman, Institute of Scientific Research in Vedas (I-SERVE), Hyderabad, India and Shri K.V.R.S. Murthy, former scientist IICT (CSIR), Govt. of India, Director, Research and Development, I-SERVE, for their valuable guidance and great support in developing this subject.

\section{References}

[1] S. Bethke and G.P. Salam. Quantum chromodynamics. K.A. Olive et al. (Particle Data Group), Chin. Phys. C, 38, 090001 (2014) and 2015 update.

[2] David J. Gross. Twenty Five Years of Asymptotic Freedom. Nucl.Phys.Proc.Suppl. $74 \quad$ (1999) 426-446 https://doi.org/10.1016/S0920-5632(99)00208-X.

[3] Weizsäcker, Carl Friedrich von, on the theory of nuclear masses; Journal of Physics 96 (1935) pages 431- 458.

[4] W. D. Myers et al. Table of Nuclear Masses according to the 1994 Thomas-Fermi Model.(from nsdssd.lbl.gov)

[5] P. Roy Chowdhury et al. Modified Bethe-Weizsacker mass formula with isotonic shift and new driplines. Modern Physics Letters A 20.21 1605-1618 (2005)

[6] J.A. Maruhn et al., Simple Models of Many-Fermion Systems, Springer-Verlag Berlin Heidelberg 2010. Chapter 2, page: 45-70. https://doi.org/10.1007/978-3-642-03839-6_2.

[7] Seshavatharam U. V. S, Lakshminarayana, S., Understanding nuclear binding energy with strong interaction. To be appeared in Physical Science International Journal.

[8] Seshavatharam U. V. S, Lakshminarayana, S., Simplified Form of the Semi-empirical Mass Formula. Prespacetime Journal, Volume 8, Issue 7, pp.881-810 (2017)

[9] Seshavatharam U. V. S, Lakshminarayana, S., On the role of strong coupling constant and nucleons in understanding nuclear stability and binding energy. Journal of Nuclear Sciences, Vol. 4, No.1, 7-18, (July 2017)

[10] N. Ghahramany et al. A new approach to nuclear binding energy in integrated nuclear model.T.8, No 2 (165). 169-181. (2011)
[11] Ghahramany et al. New approach to nuclear binding energy in integrated nuclear model. Journal of Theoretical and Applied Physics 2012, 6:3 https://doi.org/10.1186/2251-7235-6-3.

[12] N. Ghahramany et al. Stability and Mass Parabola in Integrated Nuclear Model. Universal Journal of Physics and Application 1(1): 18$25,(2013)$. 